\title{
Molecular Cloning and Tissue Distribution of Troponin I from the Japanese Pearl Oyster, Pinctada fucata
}

\author{
Daisuke Funabara*, Yoshinori Urakawa, Satoshi Kanoh \\ Graduate School of Bioresources, Mie University, Tsu, Japan \\ Email: *funabara@bio.mie-u.ac.jp
}

How to cite this paper: Funabara, D., Urakawa, Y. and Kanoh, S. (2019) Molecular Cloning and Tissue Distribution of Troponin I from the Japanese Pearl Oyster, Pinctada fucata. American Journal of Molecular Biology, 9, 29-40.

https://doi.org/10.4236/ajmb.2019.92003

Received: February 11, 2019

Accepted: March 2, 2019

Published: March 5, 2019

Copyright () 2019 by author(s) and Scientific Research Publishing Inc. This work is licensed under the Creative Commons Attribution International License (CC BY 4.0).

http://creativecommons.org/licenses/by/4.0/

\begin{abstract}
Troponin is a complex of three proteins (troponin I, troponin C, and troponin $\mathrm{T}$ ) that binds $\mathrm{Ca}^{2+}$ and is a thin filament-associated regulator of vertebrate striated muscle contraction. The function of troponin I (TnI) in vertebrates has been extensively characterized, but its role in molluscan muscles has not yet been elucidated. Our previous work suggested that the troponin $C$ subunit has a role in adductor phasic muscle but not in catch muscle. Here, we investigated the molecular characteristics of TnI from the bivalve Japanese pearl oyster, Pinctada fucata to aid the elucidation of the function of molluscan muscle troponin. We determined the primary structure of the full-length TnI protein from the $P$. fucata adductor muscle (Pifuc-TnI) and found that it is composed of 286 amino acid residues with a predicted molecular weight of 33,737. Motif structure predictions and multiple sequence alignments revealed that Pifuc-TnI has a 138 residue extension at its $\mathrm{N}$-terminus compared with rabbit TnI. This is analogous to characterized TnIs from other mollusks. However, unlike scallop TnI, Pifuc-TnI is predicted to contain two cAMP-dependent protein kinase phosphorylation sites, at residues 39 - 45 (RRGTEDD) and 145 - 151 (KKKSKRK). Phylogenetic analysis indicated that Pifuc-TnI and molluscan TnIs were grouped into the same clade. Pifuc-TnI gene structure predictions using Splign alignment of our obtained cDNA and genome sequences indicated that Pifuc-TnI consists of fifteen exons, with the start and stop codons located in exon 2 and exon 11, respectively. Using quantitative real-time PCR, we determined that the Pifuc-TnI gene is predominantly expressed in adductor phasic muscle, weakly in adductor catch muscle, and is not expressed in the gill, mantle or foot. These findings suggest that $\mathrm{TnI}$, as a component of the troponin complex, plays a regulatory role in adductor phasic muscle contraction, but not in catch contraction.
\end{abstract}




\section{Keywords}

Adductor Muscle, Catch Contraction, Pinctada fucata, Troponin, Troponin I

\section{Introduction}

Mollusk bivalve adductor muscles are composed of two muscle types: phasic and catch. Phasic muscle is used for the quick closure of shells, whereas catch muscle is involved in the sustainable closure of shells. The contraction of both muscles is regulated by intracellular $\mathrm{Ca}^{2+}$ concentrations [1]. Mollusks employ a thick filament-linked regulatory system where myosin directly binds $\mathrm{Ca}^{2+}$, leading to its activation and subsequent interaction with actin. Following a decrease in the intracellular $\mathrm{Ca}^{2+}$ concentration, myosin is inactivated, and its interaction with actin in phasic muscle is abolished. Once $\mathrm{Ca}^{2+}$ concentrations decrease to resting levels, catch muscle enters the high-tension catch state, which is able to be maintained for long periods of time. Twitchin, a giant myosin-associated protein, is thought to tether together the thin and thick filaments through its phosphorylation sites [2] [3] [4].

In contrast to molluscan muscles, vertebrate striated muscles employ a thin filament-linked regulatory system. Troponin (Tn) is the regulator of skeletal muscle contraction. It is distributed on thin filaments and inhibits the interaction between actin and myosin. Tn consists of three subunits: troponin $\mathrm{C}(\mathrm{TnC})$, troponin I (TnI), and troponin $\mathrm{T}(\mathrm{TnT})$. The binding of $\mathrm{Ca}^{2+}$ to $\mathrm{TnC}$ induces a conformational change in the troponin complex structure and enables myosin to interact with actin [5] [6] [7] [8]. Although Tn is also located in molluscan muscles, it is currently unclear whether it is involved in a similar thin filament-linked regulatory system to that in vertebrates.

The Japanese pearl oyster, Pinctada fucata, is one of the most important molluscan species in the pearl culture industry. A genome database of Pinctada fucata has recently been released and all the major muscle protein genes have been registered [9] [10] [11]. Therefore, we have used $P$. fucata as a model system to elucidate the molluscan muscle regulatory system. Our studies have focused on the adductor muscle Tn subunits to investigate the molecular mechanism of thin filament-linked regulation of molluscan muscle contraction. To this end, we recently characterized $P$. fucata TnC (Pifuc-TnC) [12] [13]. Pifuc-TnC has four EF-hand motifs, sites I-IV, and, like other molluscan TnCs [14] [15] [16], only site IV is able to bind $\mathrm{Ca}^{2+}$ [12]. A conformational change is induced in Pifuc-TnC upon $\mathrm{Ca}^{2+}$-binding [12]. A predicted three-dimensional model of Pifuc-TnC closely resembled that of vertebrate TnC, apart from a short loop (four amino acids) in the former structure that breaks the $\alpha$-helix connecting the $\mathrm{N}$ and C-terminal lobes [13]. Quantitative real-time PCR indicated that the Pifuc- $\operatorname{Tn} C$ gene is predominantly expressed in adductor phasic muscle, suggesting a role for Tn in adductor phasic muscle regulation [13]. Furthermore, recent 
proteomic and transcriptomic analyses have revealed that scallop phasic and catch muscles have different gene expression patterns [17]. Together, these findings suggest that adductor phasic and catch muscles employ different regulatory systems.

The interaction between $\mathrm{TnC}$ and $\mathrm{TnI}$ in the vertebrate troponin complex is dependent upon $\mathrm{Ca}^{2+}$ binding to TnC [18] [19] [20] [21]. Characterized TnIs from other scallop species have been found to have a long ( $>130$ residues) $\mathrm{N}$-terminal extending region that is absent in vertebrate striated muscle TnI [22] [23]. A binding analysis study using recombinant scallop TnI domains revealed that an N-terminal peptide (residues 130 - 252) that lacks the extension but contains a region homologous to residues $1-30$ of rabbit $\mathrm{TnI}$ is able to bind to both TnC and actin [23]. However, little is known about molluscan TnIs and further studies are required to unveil the function of $\mathrm{Tn}$ in the regulation of molluscan muscle contraction.

Our research is focused on the elucidation of Tn function in molluscan muscle contraction. To perform a molecular characterization of $P$. fucata TnI (Pifuc-TnI), we analyzed the full nucleotide sequence, the gene structure, and $P i$ fuc-TnI tissue expression patterns.

\section{Materials and Methods}

\subsection{Pearl Oysters}

We obtained live specimens of the Japanese pearl oyster, Pinctada fucata that were cultured in Ago Bay, Mie Prefecture, Japan. The adductor muscle, gill, mantle, and foot were dissected from each oyster body, immediately frozen in liquid nitrogen, and stored at $-80^{\circ} \mathrm{C}$ until use.

\section{2. cDNA Cloning of Pinctada fucata Troponin I}

Total RNA was extracted from the phasic part of the Pinctada fucata adductor muscle using a conventional method [24]. The partial nucleotide sequences of the $P$. fucata troponin I (Pifuc- $T n I$ ), as determined by 3 ' rapid amplification of cDNA ends (RACE), have been previously reported [10]. To determine the full-length sequence of Pifuc-TnI, 5'RACE was carried out using the 5'RACE system for Rapid Amplification of cDNA Ends, version 2.0 (Invitrogen, Carlsbad, CA, USA) using total RNA as a template. Primers were designed using the elucidated sequences of Pifuc-TnI: 5 'GCCATATGAGCTCACTGAAGGT-3' for synthesizing cDNA, 5'-TCCTCGAGTTATCTACTATGTT-3' for the first PCR, and 5'-AAGAATAGCTCGAGGATCCGG-3' for the second PCR. PCR was carried out using SapphireAmp Fast PCR Master Mix (TaKaRa Bio, Otsu, Japan) with the forward primers detailed above and the reverse primers included in the kit. The PCR conditions were as follows: 30 cycles of denaturation at $98^{\circ} \mathrm{C}$ for 5 $\mathrm{s}$, annealing at $55^{\circ} \mathrm{C}$ for $5 \mathrm{~s}$, and elongation at $72^{\circ} \mathrm{C}$ for $10 \mathrm{~s}$. The amplified DNA fragment was sequenced after insertion into a pTAC-1 vector. The determined nucleotide sequence data were registered in the DDBJ/EMBL/GenBank sequence database (accession number LC458449). 


\subsection{Comparison of the Primary Structure of $P$. fucata Troponin I with Those from Other Species}

The Pifuc-TnI primary structure was determined from the Pifuc-TnI cDNA nucleotide sequence. ClustalW (https://www.ddbj.nig.ac.jp/index-e.html) was used to compare this sequence with Troponin I amino acid sequences from other molluscan species: Japanese scallop, Chlamys nipponensis (AB206837); Yesso scallop, Mizuhopecten yessoensis (AB008005, AB008006); and rabbit, Oryctolagus cuniculus (S61403).

\subsection{Prediction of Structural Motifs in P. fucata Troponin I}

The motif structure of Pifuc-TnI was predicted by SMART

(http://smart.embl-heidelberg.de/), Pfam (http://pfam.xfam.org/), and the Eukaryotic Linear Motif resource (http://elm.eu.org/).

\subsection{Phylogenetic Analysis of Troponin I}

Phylogenetic analysis was carried out using the primary structures of troponin I from various species following sequence alignment using the ClustalW algorithm. The sequences used were: human, Homo sapiens (AAA19813.1); mouse, Mus musculus (AAA40485.1); rabbit, Oryctolagus cuniculus (AAB26824.1); rat, Rattus norvegicus (AAA42294.1); pig, Sus scrofa (AAP37479.1); common quail, Coturnix coturnix (AAB00122.1); chicken, Gallus gallus (AAA61952.1, NP_998735.1); tropical clawed frog, Xenopus tropicalis (AAH61268.1, AAH84508.1); African clawed frog, Xenopus laevis (AAA65727.1, AAL86906.1, NP_001081378.1, NP_001079556.1, NP_001079781.1); Atlantic herring, Clupea harengus (AAB05825.1); Orange-spotted grouper, Epinephelus coioides (ADG29132.1); rainbow trout, Oncorhynchus mykiss (NP_001123462.1); Atlantic salmon, Salmo salar (NP_001117133.1); mandarin fish, Siniperca chuatsi (ACM07327.1); honey bee, Apis mellifera (NP_001035346.1); domestic silkworm, Bombyx mori (NP_001037295.1); fruit fly, Drosophila melanogaster (CAA42020.1); beet webworm, Loxostege sticticalis (ABY56688.1); Japanese scallop, Chlamys nipponensis (BAE43657.1, BAE43658.1); akazara scallop, Chlamys nipponensis akazara (BAA23775.1) and Yesso scallop, Mizuhopecten yessoensis (BAA22852.1, BAA22853.1).

\subsection{Gene Structure of $P$. fucata Troponin I}

The genome sequence including the Pifuc-TnI gene was obtained by BLAST searching the Pifuc-TnI nucleotide sequence against the $P$. fucata genome database [9]. The gene structure of the Pifuc-TnI gene was predicted by analyzing cDNA and genome sequences using the Splign alignment tool (NCBI) [25].

\subsection{Gene Expression Analysis of $P$. fucata Troponin I in Tissues}

Gene expression patterns of Pifuc-TnI in the catch and phasic muscles, gill, mantle, and foot were analyzed by quantitative real-time PCR. cDNAs were 
synthesized using total RNA from each tissue as templates using RiverTra Ace ${ }^{\infty}$ qPCR RT Master Mix (Toyobo Co. Ltd., Osaka, Japan). The primers and a probe were designed by the Universal Probe Library Assay Design Center (Roche Diagnostics, Mannheim, Germany) using the full-length nucleotide sequence determined in this study. The primers used were:

5 'AGCTGAAGAGGAAACCTCCAC-3' (forward) and

5 'TGGCTATATCGTCCTCAGTGC-3' (reverse). Probe \#18 (Roche Diagnostics) was used as a TaqMan probe. P. fucata $\beta$-actin (AF378128) was used as an internal standard. The primers used for $\beta$-actin were:

5 '-TCGTTCCTCGGAATGGAA-3' (forward) and

5 '-TCGACATCGCATTTGAGAAT-3' (reverse). Probe \#151 (Roche Diagnostics) was used as a TaqMan probe. The PCR reaction was performed using Eagle Taq Master Mix with ROX (Roche Diagnostics).

\section{Results}

\subsection{Molecular Characteristics of $P$. fucata Troponin I}

The full-length nucleotide sequence of Pifuc-TnI was obtained through cDNA cloning and was found to contain 3169 nucleotides (nt), which includes an open reading frame of $861 \mathrm{nt}$, a $432 \mathrm{nt} 5$ 'untranslated region, and a $1936 \mathrm{nt} 3$ 'untranslated region (Figure 1). It encodes a protein of 286 amino acid residues in length with a predicted molecular weight of 33,737 (Figure 1). The amino acid sequence of Pifuc-TnI was 50\% homologous to both Japanese scallop and Yesso scallop TnIs and was $21 \%$ homologous to rabbit TnI (Figure 2). Analogous to known scallop TnIs, Pifuc-TnI has an N-terminal sequence extension of 136 residues compared with rabbit skeletal TnI [22] [23]. This extension is rich in the charged amino acids, glutamic acid and arginine. Motif structure prediction software indicated that Pifuc-TnI contains two coiled-coils (at residues 4 - 25 and 60 - 152) and a troponin motif (residues 161 - 286) (Figure 3). In contrast to

scallop TnIs, Pifuc-TnI also contains consensus sequences for cAMP-dependent protein kinases (PKAs), which are located at residues $39-45$ (RRGTEDD) and 145 - 151 (KKKSKRK) (Figure 2 and Figure 3).

BLAST searching of Pifuc-TnI against the $P$. fucata genome database yielded two gene models (pfu_aug2.0_433.1_10766.t1 and pfu_aug2.0_433.1_10766.t2) that were computationally predicted from the genome sequence (data not shown). However, neither were found to exactly match our obtained Pifuc-TnI sequence. This suggests the possibility that some TnI isoforms are expressed from a single gene by alternative RNA processing; however, our investigations did not identify any Pifuc-TnI isoform encoding sequences.

Phylogenetic analysis indicated that Pifuc-TnI and molluscan TnIs were grouped into the same clade (Figure 4).

\subsection{Gene Structure of $P$. fucata Troponin I}

BLAST searching of our obtained Pifuc-TnI nucleotide sequence against the genome database of $P$. fucata yielded a single nucleotide sequence at scaffold 433.1. 


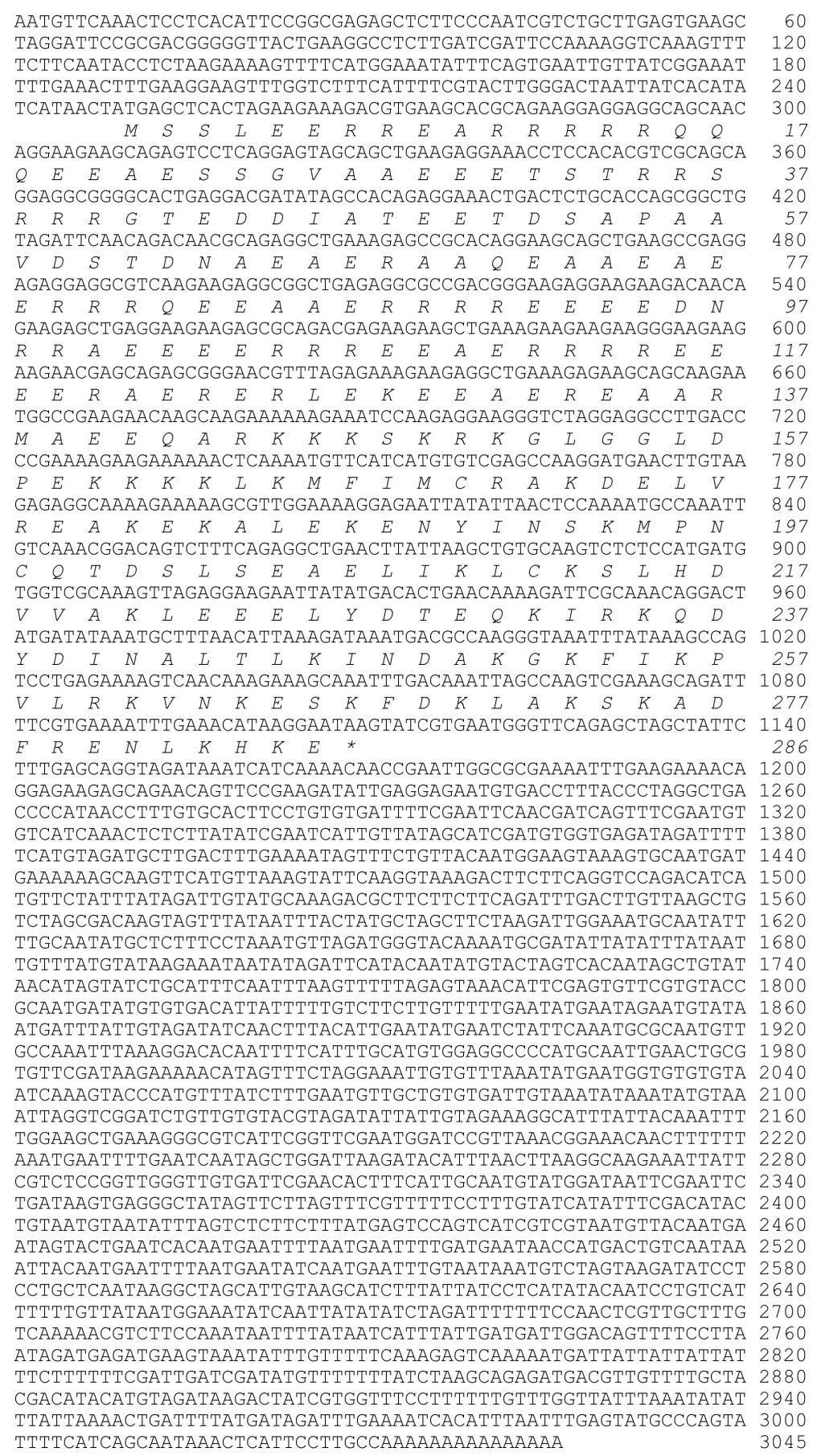

Figure 1. Nucleotide and deduced amino acid sequences of Pinctada fucata troponin I. The amino acid sequence is represented in italics. The numbers on the right of the sequences denote nucleotides from the 5 '-end and amino acid residues from the $\mathrm{N}$-terminus. The stop codon is indicated by an asterisk.

We then predicted the gene structure of Pifuc-TnI using Splign alignment of the obtained cDNA and genome sequences. Pifuc-TnI consists of fifteen exons (Figure 5), and the start and stop codons are located in exon 2 and exon 11, respectively. 


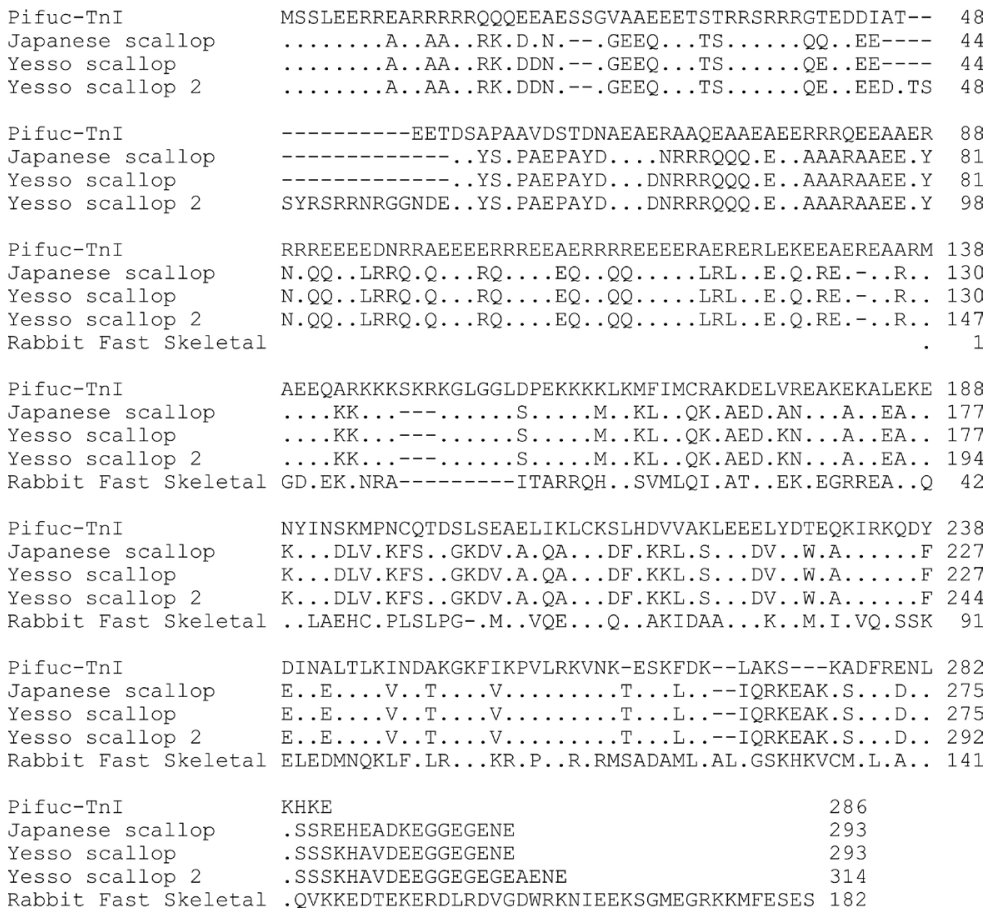

Figure 2. Multiple sequence alignments of the determined Pinctada fucata troponin I amino acid sequence to troponin I proteins from other species. Compared sequences were obtained from the DDBJ database: Japanese scallop Chlamys nipponensis (AB206837), Yesso scallop Mizuhopecten yessoensis (AB008005, AB008006), and rabbit Oryctolagus cuniculus (S61403). Numbers on the right represent the number of amino acid residues from the $\mathrm{N}$-terminus. Gaps were inserted to optimize the sequence alignments. Dots indicate the identical residues present in both Pifuc-TnI and a particular compared sequence.

\subsection{Distribution of Troponin I in $P$. fucata Tissues}

Pifuc-TnI was predominantly expressed in adductor phasic muscle, while weak expression was detected in adductor catch muscle, and no expression was detected in the gill, mantle, or foot (Figure 6). Therefore, the tissue distribution of Pifuc-TnI is equivalent to that of Pifuc-TnC [13]. These findings suggest that Tn is involved in the regulation of the phasic adductor muscle, rather than the catch adductor muscle.

\section{Discussion}

In this study, we have analyzed the molecular characteristics of Pifuc-TnI. As the primary sequence of Pifuc-TnI is $50 \%$ homologous to scallop TnI, it is possible that their steric structures are similar and they form Tn complexes in an analogous manner. Recombinant peptides of scallop and rabbit TnIs have been used to analyze the function of scallop TnI [23]. A scallop TnI C-terminal peptide (residues 232 - 292) was able to bind to actin-tropomyosin and inhibit actomyosin-tropomyosin Mg-ATPase, but was unable to interact with TnC. However, an $\mathrm{N}$-terminal scallop peptide that contains a region homologous to residues 
Pifuc-Tnl (286 aa)

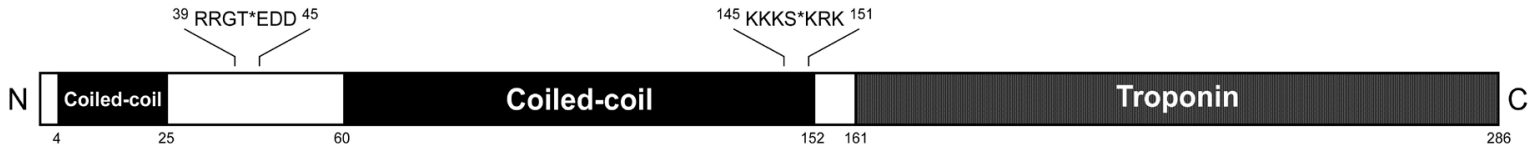

Japanese scallop Tnl (293 aa)

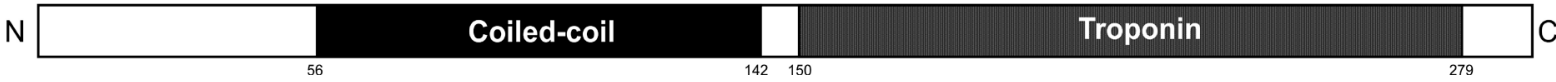

Figure 3. Motif structures of molluscan troponin I. Schematic representations of TnIs from Pinctada fucata and Japanese scallop Chlamys nipponensis showing the positions of predicted motif structures predicted by SMART, Pfam, and the Eukaryotic Linear Motif resource. Numbers represent the relative amino acid positions of each motif (from the N-terminus). RRGT*EDD and $\mathrm{KKKS}^{\star} \mathrm{KRK}$ are the potential PKA phosphorylation sites in Pifuc-TnI. Asterisks indicate the phosphorylatable threonine and serine residues in each consensus sequence.

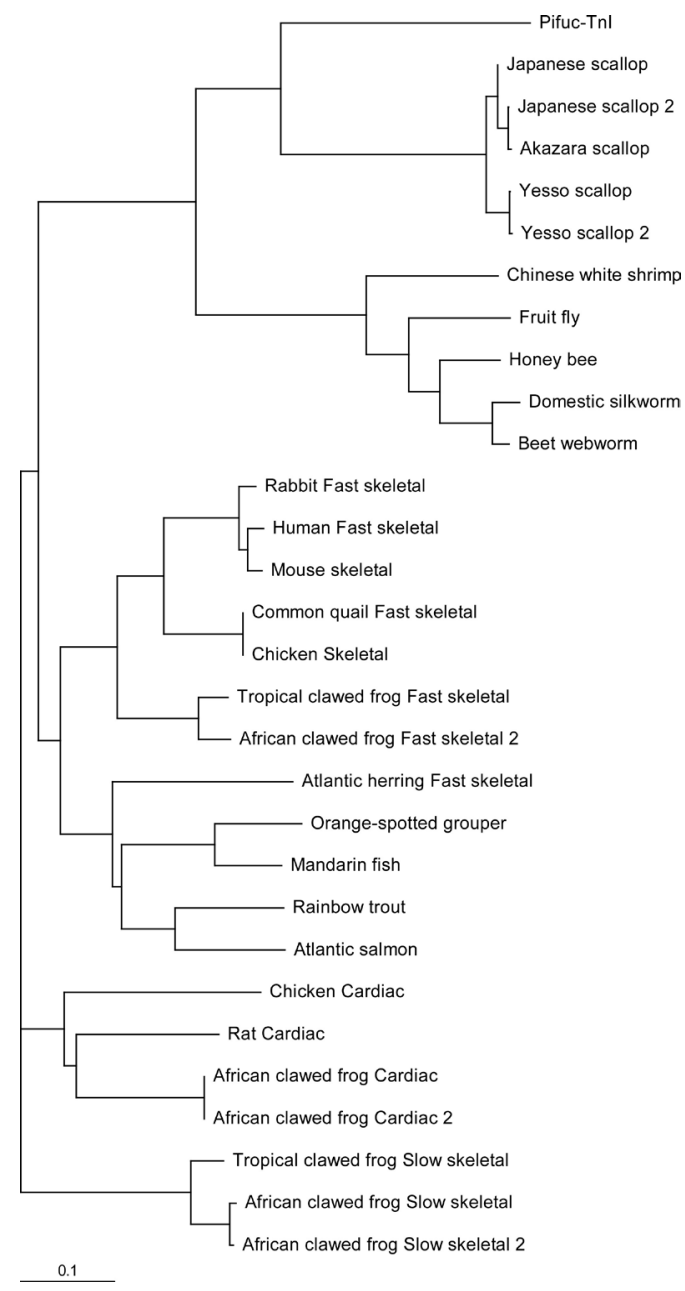

Figure 4. Phylogenetic tree showing the relationship among the troponin I amino acid sequences from Pinctada fucata and other species. The tree was generated using the ClustalW algorithm. The GenBank accession numbers of each sequence used in the analysis are listed in Materials and Methods. 


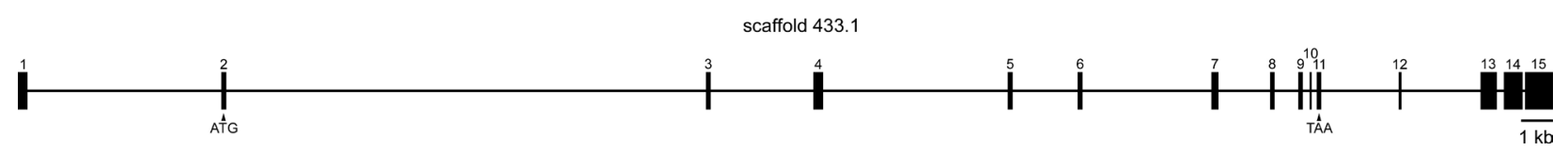

Figure 5. Gene structure of Pinctada fucata troponin I. Black boxes indicate exons, and intervening lines represent introns. The ATG start and TAA stop codons are indicated.

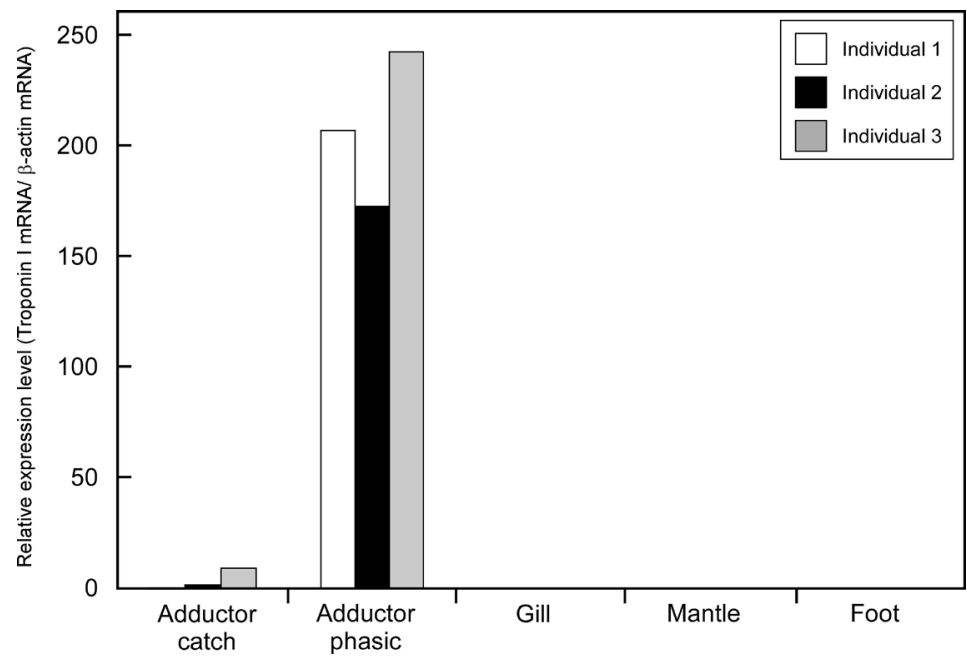

Figure 6. Gene expression patterns of troponin I in Pinctada fucata tissues. Quantitative real-time PCR analysis was performed to examine troponin I gene expression in Pinctada fucata adductor catch muscle, adductor phasic muscle, gill, mantle, and foot. The data shown are representative of three independent experiments. The $y$-axis indicates relative troponin I expression levels using $\beta$-actin as an internal standard.

1 - 30 of rabbit TnI strongly bound both $\mathrm{TnC}$ and actin and was able to activate the actomyosin-tropomyosin $\mathrm{Mg}$-ATPase in a $\mathrm{Ca}^{2+}$-dependent manner. As the C-terminal regions of Pifuc-TnI and scallop TnI are relatively homologous (Figure 2), we predict that the Pifuc-TnI C-terminus also does not play a role in the regulation of muscle contraction [23]. Therefore, molluscan TnI is predicted to regulate muscle contraction through an activation activity that is exerted by its $\mathrm{N}$-terminal region. However, the $\mathrm{N}$-terminal extending region of scallop $\mathrm{TnI}$ (that is not present in rabbit skeletal TnI) was shown to be unnecessary for this function and, instead is thought to play a role in maintaining the structural integrity of the Tn complex. Further studies are required to completely elucidate the role of this domain in Tn function.

TnI has been shown to be phosphorylated by several different protein kinases in vertebrate cardiac muscles [26]. We found potential PKA consensus sequences in Pifuc-TnI at residues 39 - 45 (RRGTEDD) and 145 - 151 (KKKSKRK). Intriguingly, scallop TnI does not contain any predicted PKA consensus sequences (Figure 3). In Pifuc-TnI, one potential phosphorylation site is located in the linker region between the two coiled-coil motifs and the other is located at the end of the second coiled-coil motif. It is possible that phosphorylation of these sites could induce a conformational change in the Pifuc- TnC molecule that al- 
ters the interaction between TnI, TnT and TnC. Site-specific phosphorylation of cardiac TnI by different kinases has been revealed as a key physiological mechanism for the modulation of myofilament properties [26]. It is possible that Pifuc-Tn regulates adductor phasic muscle contraction in a similar way. PKA phosphorylation of molluscan catch muscle twitchin has been shown to abolish the catch state to relax the muscle [1]. Interestingly, PKA is able to phosphorylate twitchin proteins isolated from both scallop phasic and catch muscles in vitro, suggesting that both muscles utilize a signaling pathway that is dependent upon PKA phosphorylation [27]. However, scallop TnI contains no PKA typical consensus sequences. It is certainly possible that scallop and pearl oyster adductor muscles employ different molecular mechanisms for muscle regulation. Further studies on Pifuc-TnI, particularly investigations of the effect of PKA phosphorylation on its function, are required to reveal the role of troponin in Pinctada fucata.

\section{Acknowledgements}

This study was supported by JSPS KAKENHI Grant Number JP16K07872. We thank Emma Andrew, PhD, from Edanz Group (http://www.edanzediting.com/ac) for editing a draft of this manuscript.

\section{Conflicts of Interest}

The authors declare no conflicts of interest regarding the publication of this paper.

\section{References}

[1] Funabara, D., Kanoh, S., Siegman, M.J., Butler, T.M., Hartshorne, D.J. and Watabe, S. (2005) Twitchin as a Regulator of Catch Contraction in Molluscan Smooth Muscle. Journal of Muscle Research and Cell Motility, 26, 455-460. https://doi.org/10.1007/s10974-005-9029-2

[2] Funabara, D., Hamamoto, C., Yamamoto, K., Inoue, A., Ueda, M., Osawa, R., Kanoh, S., Hartshorne, D.J., Suzuki, S. and Watabe, S. (2007) Unphosphorylated Twitchin Forms a Complex with Actin and Myosin That May Contribute to Tension Maintenance in Catch. Journal of Experimental Biology, 210, 4399-4410. https://doi.org/10.1242/jeb.008722

[3] Funabara, D., Osawa, R., Ueda, M., Kanoh, S., Hartshorne, D.J. and Watabe, S. (2009) Myosin Loop 2 Is Involved in the Formation of a Trimeric Complex of Twitchin, Actin, and Myosin. The Journal of Biological Chemistry, 284, 18015-18020. https://doi.org/10.1074/jbc.M109.016485

[4] Butler, T.M., Mooers, S.U., Narayan, S.R. and Siegman, M.J. (2010) The N-Terminal Region of Twitchin Binds Thick and Thin Contractile Filaments: Redundant Mechanisms of Catch Force Maintenance. The Journal of Biological Chemistry, 285 40654-40665. https://doi.org/10.1074/jbc.M110.166041

[5] Leavis, P.C., Gergely, J. and Szent-Gyorgyi, A.G. (1984) Thin Filament Proteins and Thin Filament-Linked Regulation of Vertebrate Muscle Contraction. Critical Reviews in Biochemistry, 16, 235-305. https://doi.org/10.3109/10409238409108717

[6] Ohtsuki, I., Maruyama, K. and Ebashi, S. (1986) Regulatory and Cytoskeletal Pro- 
teins of Vertebrate Skeletal Muscle. Advances in Protein Chemistry, 38, 1-67. https://doi.org/10.1016/S0065-3233(08)60525-2

[7] Zot, A.S. and Potter, J.D. (1987) Structural Aspects of Troponin-Tropomyosin Regulation of Skeletal Muscle Contraction. Annual Review of Biophysics and Biophysical Chemistry, 16, 535-559. https://doi.org/10.1146/annurev.bb.16.060187.002535

[8] Farah, C.S. and Reinach, F.C. (1995) The Troponin Complex and Regulation of Muscle Contraction. FASEB Journal, 9, 755-767. https://doi.org/10.1096/fasebj.9.9.7601340

[9] Takeuchi, T., Kawashima, T., Koyanagi, R., Gyoja, F., Tanaka, M., Ikuta, T., Shoguchi, E., Fujiwara, M., Shinzato, C., Hisata, K., Fujie, M., Usami, T., Nagai, K., Maeyama, K., Okamoto, K., Aoki, H., Ishikawa, T., Masaoka, T., Fujiwara, A., Endo, K., Endo, H., Nagasawa, H., Kinoshita, S., Asakawa, S., Watabe, S. and Satoh, N. (2012) Draft Genome of the Pearl Oyster Pinctada fucata: A Platform for Understanding Bivalve Biology. DNA Research, 19, 117-130.

https://doi.org/10.1093/dnares/dss005

[10] Funabara, D., Watanabe, D., Satoh, N. and Kanoh, S. (2013) Genome-Wide Survey of Genes Encoding Muscle Proteins in the Pearl Oyster, Pinctada fucata. Zoological Science, 30, 817-825. https://doi.org/10.2108/zsj.30.817

[11] Takeuchi, T., Koyanagi, R., Gyoja, F., Kanda, M., Hisata, K., Fujie, M., Goto, H., Yamasaki, S., Nagai, K., Morino, Y., Miyamoto, H., Endo, K., Endo, H., Nagasawa, H., Kinoshita, S., Asakawa, S., Watabe, S., Satoh, N. and Kawashima, T. (2016) Bivalve-Specific Gene Expansion in the Pearl Oyster Genome: Implications of Adaptation to a Sessile Lifestyle. Zoological Letters, 2, 3.

https://doi.org/10.1186/s40851-016-0039-2

[12] Funabara, D., Ishikawa, D., Urakawa, Y. and Kanoh, S. (2018) $\mathrm{Ca}^{2+}$-Induced Conformational Change of Troponin C from the Japanese Pearl Oyster, Pinctada fucata. American Journal of Molecular Biology, 8, 205-214. https://doi.org/10.4236/ajmb.2018.84018

[13] Funabara, D., Urakawa, Y. and Kanoh, S. (2018) Molecular Cloning and Tissue Distribution of Troponin C from the Japanese Pearl Oyster, Pinctada fucata. American Journal of Molecular Biology, 8, 166-177. https://doi.org/10.4236/ajmb.2018.83014

[14] Nishita, K., Tanaka, H. and Ojima, T. (1994) Amino Acid Sequence of Troponin C from Scallop Striated Adductor Muscle. Journal of Biological Chemistry, 269, 3464-3468.

[15] Ojima, T., Tanaka, H. and Nishita, K. (1994) Cloning and Sequence of a cDNA Encoding Akazara Scallop Troponin C. Archives of Biochemistry and Biophysics, 311, 272-276. https://doi.org/10.1006/abbi.1994.1237

[16] Ojima, T., Ohta, T. and Nishita, K. (2001) Amino Acid Sequence of Squid Troponin C. Comparative Biochemistry and Physiology Part B: Biochemistry and Molecular Biology, 129, 787-796. https://doi.org/10.1016/S1096-4959(01)00397-9

[17] Sun, X., Liu, Z., Wu, B., Zhou, L., Wang, Q., Wu, W. and Yang, A. (2018) Differences between Fast and Slow Muscles in Scallops Revealed through Proteomics and Transcriptomics. BMC Genomics, 19, 377. https://doi.org/10.1186/s12864-018-4770-2

[18] Syska, H., Wilkinson, J.M., Grand, R.J. and Perry, S.V. (1976) The Relationship between Biological Activity and Primary Structure of Troponin I from White Skeletal Muscle of the Rabbit. Biochemical Journal, 153, 375-387. https://doi.org/10.1042/bj1530375 
[19] Ngai, S.-M. and Hodges, R.S. (2001) Characterization of the Biologically Important Interaction between Troponin $\mathrm{C}$ and the N-Terminal Region of Troponin I. Journal of Cellular Biochemistry, 83, 99-110. https://doi.org/10.1002/jcb.1212

[20] Ngai, S.-M. and Hodges, R.S. (2001) Structural and Functional Studies on Troponin I and Troponin C Interactions. Journal of Cellular Biochemistry, 83, 33-46. https://doi.org/10.1002/jcb.1204

[21] Tripet, B., Van Eyk, J.E. and Hodges, R.S. (1997) Mapping of a Second Actin-Tropomyosin and a Second Troponin C Binding Site within the C Terminus of Troponin I, and Their Importance in the $\mathrm{Ca}^{2+}$-Dependent Regulation of Muscle Contraction. Journal of Molecular Biology, 271, 728-750. https://doi.org/10.1006/jmbi.1997.1200

[22] Tanaka, H., Ojima, T. and Nishita, K. (1998) Amino Acid Sequence of Troponin-I from Akazara Scallop Striated Adductor Muscle. The Journal of Biochemistry, 124, 304-310. https://doi.org/10.1093/oxfordjournals.jbchem.a022112

[23] Tanaka, H., Takeya, Y., Doi, T., Yumoto, F., Tanokura, M., Ohtsuki, I., Nishita, K. and Ojima, T. (2005) Comparative Studies on the Functional Roles of N- and C-Terminal Regions of Molluskan and Vertebrate Troponin-I. The FEBS Journal, 272, 4475-4486. https://doi.org/10.1111/j.1742-4658.2005.04866.x

[24] Chomczynski, P. (1993) A Reagent for the Single-Step Simultaneous Isolation of RNA, DNA and Proteins from Cell and Tissue Samples. BioTechniques, 15, 532-537.

[25] Kapustin, Y., Souvorov, A., Tatusova, T. and Lipman, D. (2008) Splign: Algorithms for Computing Spliced Alignments with Identification of Paralogs. Biology Direct, 3, 20. https://doi.org/10.1186/1745-6150-3-20

[26] Layland, J., Solaro, R.J. and Shah, A.M. (2005) Regulation of Cardiac Contractile Function by Troponin I Phosphorylation. Cardiovascular Research, 66, 12-21. https://doi.org/10.1016/j.cardiores.2004.12.022

[27] Funabara, D., Watabe, S. and Kanoh, S. (2015) Phosphorylation Properties of Twitchin from Yesso Scallop Catch and Striated Muscles. Fisheries Science, 81, 541-550. https://doi.org/10.1007/s12562-015-0866-7 